\title{
ANTLIA B: A FAINT DWARF GALAXY MEMBER OF THE NGC 3109 ASSOCIATION
}

\author{
D. J. Sand ${ }_{1}^{1}$, K. Spekkens ${ }^{2}$, D. Crnojević ${ }^{1}$, J. R. Hargis ${ }^{3}$, B. Willman ${ }^{3}$, J. Strader ${ }^{4}$, and C. J. Grillmair ${ }^{5}$ \\ ${ }^{1}$ Physics Department, Texas Tech University, Box 41051, Lubbock, TX 79409-1051, USA; david.sand@ttu.edu \\ ${ }^{2}$ Department of Physics, Royal Military College of Canada, P.O. Box 17000, Station Forces, Kingston, Ontario, K7K 7B4, Canada \\ ${ }^{3}$ Department of Astronomy, Haverford College, 370 Lancaster Avenue, Haverford, PA 19041, USA \\ ${ }^{4}$ Department of Physics and Astronomy, Michigan State University, East Lansing, MI 48824, USA \\ ${ }^{5}$ Spitzer Science Center, 1200 E. California Boulevard, Pasadena, CA 91125, USA \\ Received 2015 August 6; accepted 2015 September 14; published 2015 October 9
}

\begin{abstract}
We report the discovery of Antlia B, a faint dwarf galaxy at a projected distance of $\sim 72 \mathrm{kpc}$ from NGC 3109 $\left(M_{V} \sim-15 \mathrm{mag}\right)$, the primary galaxy of the NGC 3109 dwarf association at the edge of the Local Group. The tip of the red giant branch distance to Antlia B is $D=1.29 \pm 0.10 \mathrm{Mpc}$, which is consistent with the distance to NGC 3109. A qualitative analysis indicates the new dwarf's stellar population has both an old, metal-poor red giant branch $(\gtrsim 10 \mathrm{Gyr},[\mathrm{Fe} / \mathrm{H}] \sim-2)$, and a younger blue population with an age of $\sim 200-400 \mathrm{Myr}$, analogous to the original Antlia dwarf, another likely satellite of NGC 3109. Antlia B has $\mathrm{H}$ I gas at a velocity of $v_{\text {helio, } \mathrm{H} \text { I }}=$ $376 \mathrm{~km} \mathrm{~s}^{-1}$, confirming the association with NGC $3109\left(v_{\text {helio }}=403 \mathrm{~km} \mathrm{~s}^{-1}\right)$. The $\mathrm{H}_{\mathrm{I}}$ gas mass $\left(M_{\mathrm{H}_{\mathrm{I}}}=2.8 \pm\right.$ $\left.0.2 \times 10^{5} M_{\odot}\right)$, stellar luminosity $\left(M_{V}=-9.7 \pm 0.6 \mathrm{mag}\right)$ and half light radius $\left(r_{\mathrm{h}}=273 \pm 29 \mathrm{pc}\right)$ are all consistent with the properties of dwarf irregular and dwarf spheroidal galaxies in the Local Volume, and is most similar to the Leo P dwarf galaxy. The discovery of Antlia B is the initial result from a Dark Energy Camera survey for halo substructure and faint dwarf companions to NGC 3109 with the goal of comparing observed substructure with expectations from the $\Lambda+$ Cold Dark Matter model in the sub-Milky Way regime.
\end{abstract}

Key words: dark matter - galaxies: dwarf

\section{INTRODUCTION}

The faint end of the galaxy luminosity function is important for understanding the astrophysics of the $\Lambda+$ Cold Dark Matter $(\Lambda \mathrm{CDM})$ picture of galaxy formation. Over the last $\sim 15$ years, observational work has uncovered a population of very faint dwarf galaxies around the Milky Way (MW; e.g., Willman 2010 and references therein) and M31 (e.g., Martin et al. 2013, among others), while many more should be discovered by upcoming surveys (e.g., Hargis et al. 2014). At the same time numerical simulations indicate that there may be hundreds more subhalos that are effectively "dark." There are many physical mechanisms that are expected to either keep small dark matter halos dark, or severely limit the number of stars they form (e.g., ultraviolet heating from reionization, supernova feedback, tidal/ram pressure stripping; see e.g., Macciò et al. 2010; Arraki et al. 2014; Peñarrubia et al. 2012; Pontzen \& Governato 2012; Bovy \& Dvorkin 2013, and many others), but it is difficult to disentangle these effects by only looking at the dwarf galaxy population in a single environment - the Local Group.

The search for faint satellites around the MW continues to flourish, most recently due to the Dark Energy Camera (DECam) at Cerro Tololo International Observatory (CTIO) and the accompanying Dark Energy Survey (DES), as well as other DECam wide-field surveys (although non-DECam surveys have also been successful, e.g., Pan-STARRS; Laevens et al. 2015a, 2015b). Since the beginning of 2015, 10 new MW dwarf satellites have been found with DECam (Bechtol et al. 2015; Kim \& Jerjen 2015; Koposov et al. 2015; Martin et al. 2015). Of particular interest has been the possible association of these new dwarf systems with the Large Magellanic Cloud (Deason et al. 2015; Koposov et al. 2015). Faint dwarfs associated with larger dwarf galaxies are expected in the $\Lambda$ CDM model (e.g., D'Onghia \& Lake 2008; Wheeler et al. 2015). However, it will be difficult to definitively associate a given satellite galaxy with the Magellanic Cloud system as opposed to the MW. Searching for faint dwarf galaxy systems around nearby, isolated dwarf galaxy groups is a more promising route to understanding the role that parent galaxy mass plays in determining faint satellite properties.

Motivated to push near-field cosmology beyond the Local Group, we have completed a wide-field survey around NGC $3109\left(M_{V} \sim-15, D \sim 1.28 \mathrm{Mpc}\right.$; McConnachie 2012), the most prominent galaxy in the "NGC 3109 dwarf association" (Tully et al. 2006). Other members of the dwarf association include the Antlia dwarf, Sextans A and B, and possibly the recently discovered Leo P (e.g., Giovanelli et al. 2013). Undertaken with DECam at CTIO, our goals were to find further faint satellite companions to NGC 3109 and to study the structure and metallicity of its halo. The complete dataset consists of 15 DECam pointings $\left(45 \mathrm{deg}^{2}\right)$, giving partial coverage out to $R \sim 100 \mathrm{kpc}$ from NGC 3109's center. Typical image depths are $r \sim 24.5 \mathrm{mag}$, which is $\sim 2$ mag below the tip of the red giant branch (TRGB) at $D \sim 1.3 \mathrm{Mpc}$.

During inspection of the incoming data at the telescope, we spotted a new dwarf galaxy companion to NGC 3109, which we have dubbed Antlia B (Figure 1), corresponding to its constellation and the prior existence of the Antlia dwarf. Here we present this new discovery. Future work will present a resolved stellar analysis of the halo of NGC 3109, as well as Hubble Space Telescope (HST) imaging of Antlia B and other faint dwarf galaxy candidates (PID: 14078; PI: J. Hargis). In Section 2 we present our observational dataset, while in Section 3 we present our physical measurements of Antlia B. We discuss Antlia B in context and conclude in Section 4. 

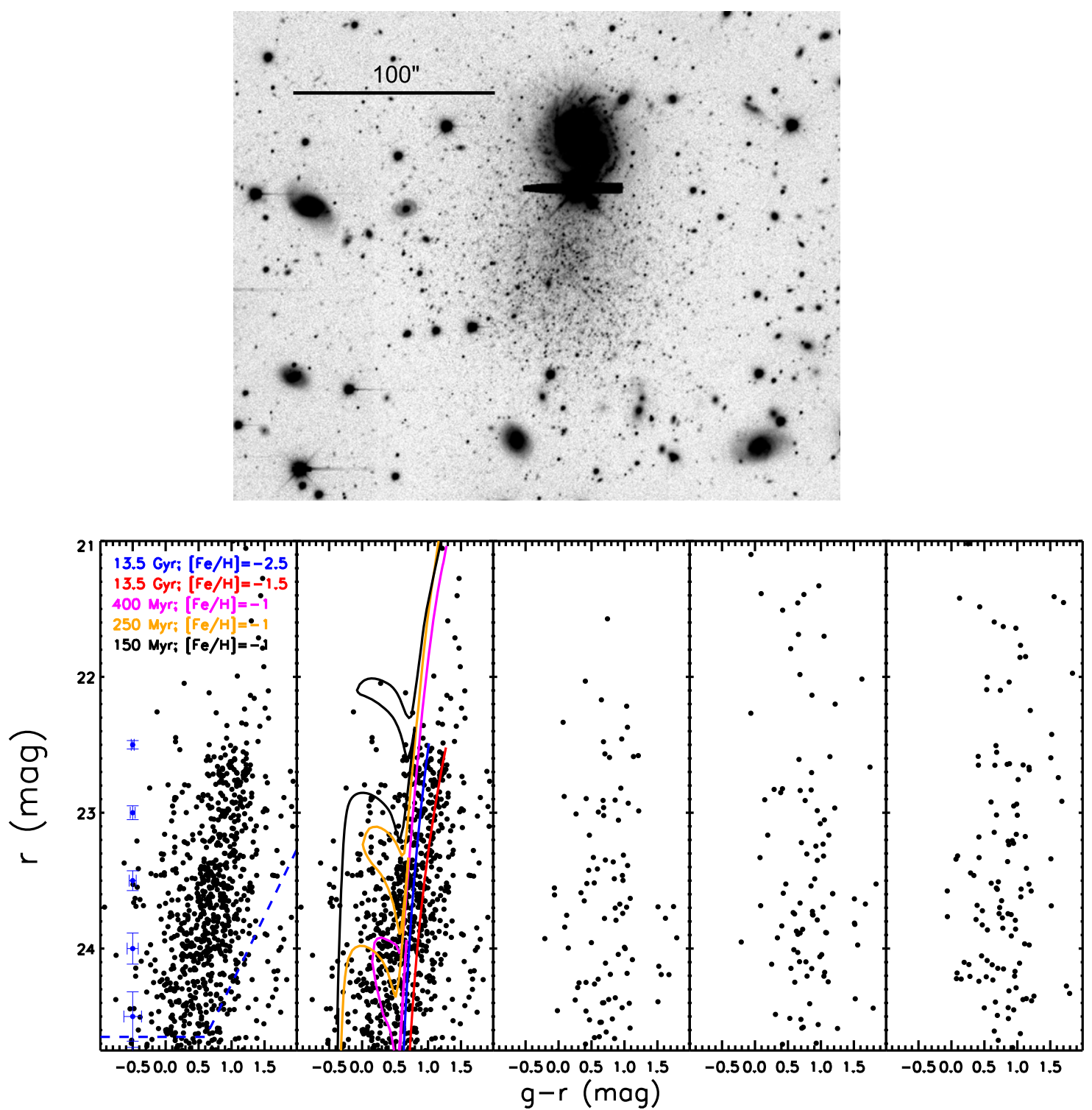

Figure 1. Top: DECam $r$-band image of Antlia B. Note the saturated foreground star and background spiral galaxy which partially overlaps Antlia B in the northern direction. North is up and east is to the left. Bottom: in the two left panels, we display our CMD of Antlia B within $1 r_{\mathrm{h}}(43$ !' 2 ). Along the left side of the far left CMD are the typical uncertainties at different $r$-band magnitudes, as determined via artificial star tests. The blue dashed line shows the 50\% completeness limit. In the second panel we plot several representative theoretical isochrones, indicating that Antlia B has both an old, metal-poor stellar population $(>10 \mathrm{Gyr}$, [Fe/H] $\sim-2)$ and a younger, more metal-rich component $(\sim 200-400 \mathrm{Myr},[\mathrm{Fe} / \mathrm{H}] \sim-1)$. See Section 3.2 for a discussion. The three right panels show CMDs from random equal-area regions, illustrating typical "background" CMDs.

\section{OBSERVATIONS}

\subsection{Optical Broadband Imaging}

The broadband data presented in this paper were taken on 2015 March 13 (UT) with DECam (Flaugher et al. 2015) on the Blanco $4 \mathrm{~m}$ telescope (2015A-0130; PI: D. Crnojević). DECam has $622 \mathrm{~K} \times 4 \mathrm{~K}$ CCDs arranged in a hexagonally shaped mosaic. The pixel scale of 0 ". 27 per pixel yields a total field of view of 3 square degrees. All of the imaging data were reduced using the DECam community pipeline (Valdes et al. 2014), and we directly use the stacked, world coordinate system-corrected $g$ and $r$ band images. The final image stacks consisted of $7 \times 300 \mathrm{~s}$ and $7 \times 150 \mathrm{~s}$ images in the $g$ and $r$ bands, respectively, with image point spread functions of $\sim 0{ }^{\prime \prime} 9$ for both. Small dithers between individual exposures were used to cover the chip gaps in the final stacks.
We extracted a single "tile" containing Antlia B $\left(\sim 45^{\prime} \times 30^{\prime}\right.$ in size) for our analysis. Stellar photometry was performed using a methodology similar to previous work on Local Group/ Volume dwarfs (e.g., Sand et al. 2012, 2014) with the DAOPHOTII/AlLSTAR package (Stetson 1994).

Several SDSS fields at varying airmasses were imaged every night of our NGC 3109 campaign, and we utilized the Radiometric All-sky Infrared Camera (Lewis et al. 2010) data in the image headers to verify that the sky was clear at each position. Calibration zeropoints, linear color terms and extinction coefficients were derived from the SDSS fields in order to convert instrumental magnitudes to the SDSS photometric system, which is approximately an $\mathrm{AB}$ system. Although the entire NGC 3109 DECam dataset was not photometric, the relevant Antlia B pointing was, and so we 
applied our derived zeropoints and color terms directly to our stellar catalogs.

Once the photometry was calibrated, we performed artificial star tests (utilizing the DAOPHOT routine ADDSTAR) to determine our photometric errors and completeness. Over many iterations, we injected $\sim 5 \times 10^{5}$ artificial stars into our data, with an $r$-band magnitude range of 18-30 and a $g-r$ color of -0.5 to 2.0 , and re-analyzed these data in an identical fashion as the unaltered data. The $50 \%(90 \%)$ completeness level was at $r=24.7$ (23.6) and $g=25.3$ (24.5) mag. The data do not suffer from crowding incompleteness until $r \sim 24.5$ in the very central regions of Antlia B. For the purpose of this work, we simply derive our structural parameters for Antlia B with a magnitude cutoff of $r=24.5 \mathrm{mag}$ (along with consistency checks) so as to avoid any issues (see Section 3.3).

The final photometric catalog was corrected for Galactic extinction (Schlegel et al. 1998; Schlafly \& Finkbeiner 2011) star by star, with a typical color excess of $E(B-V) \approx 0.069$ mag. All magnitudes presented in this work have this correction applied. In Figure 1 we show the CMD of Antlia B within its derived half-light radius (Section 3.3), along with several equalarea "background" CMDs. We discuss the stellar populations in Antlia B in Section 3.2.

\section{2. $H \alpha$ Imaging}

Narrowband $\mathrm{H} \alpha$ imaging of Antlia B was obtained on 2015 June 12 (UT) with the Goodman High-throughput Spectrograph (Clemens et al. 2004) on the SOAR telescope. The observations (taken in $1 . " 5-2$ !" 0 seeing conditions) included $3 \times 300 \mathrm{~s}$ in the $\mathrm{H} \alpha$ filter $\left(\lambda_{\text {central }}=6565 \AA\right.$, $\mathrm{FWHM}=65 \AA$ ) and $4 \times 120 \mathrm{~s}$ in the $r$ band for continuum subtraction. Standard image processing and calibration steps were carried out to produce a continuum-subtracted $\mathrm{H} \alpha$ image and flux limits (Kennicutt et al. 2008). No $\mathrm{H} \alpha$ emission was detected in association with Antlia $\mathrm{B}$, to a $3 \sigma$ point source detection limit of $\sim 2.1 \times 10^{-16} \mathrm{erg} \mathrm{s}^{-1} \mathrm{~cm}^{-2}$.

\subsection{Green Bank Telescope (GBT) Observations}

We obtained director's discretionary time on the Robert C. Byrd GBT in 2015 June (Program AGBT15A_384; PI: K. Spekkens) to carry out position-switched $\mathrm{H}_{\mathrm{I}}$ observations of Antlia B. We used the Versatile GBT Astronomical Spectrometer (VEGAS) with a bandpass of $11.72 \mathrm{MHz}$ and $0.4 \mathrm{kHz}$ channels centered at $V_{\mathrm{LSR}}=500 \mathrm{~km} \mathrm{~s}^{-1}$ in the $\mathrm{H}_{\mathrm{I}}$ line to obtain spectra along the line of sight to Antlia B as well as at several reference locations. The total integration time of 35 minutes was divided equally between the on- and off-target locations, the latter serving to flatten the spectral baseline in the target spectrum at the processing stage. The 9.1 arcmin FWHM beam of the GBT at this observing frequency well exceeds the halflight stellar diameter of Antlia B $(\sim 1 ! 4$, see Section 3.2$)$, and we therefore expect any $\mathrm{H}_{\mathrm{I}}$ in this system to be recovered in a single GBT pointing.

The data were reduced using the standard GBTIDL ${ }^{6}$ routine getps and smoothed to a spectral resolution of $2 \mathrm{~km} \mathrm{~s}^{-1}$. The resulting spectrum is shown in Figure 2: we detect $\mathrm{H}$ i emission along the line of sight to Antlia $\mathrm{B}$ with $\mathrm{S} / \mathrm{N} \sim 15$. The profile peak at $v_{\text {helio }} \sim 375 \mathrm{~km} \mathrm{~s}^{-1}$ indicates that Antlia B has a very similar velocity to NGC $3109\left(v_{\text {helio, } \mathrm{H}}=403 \mathrm{~km} \mathrm{~s}^{-1}\right)$.

\footnotetext{
6 http://gbtidl.nrao.edu/
}

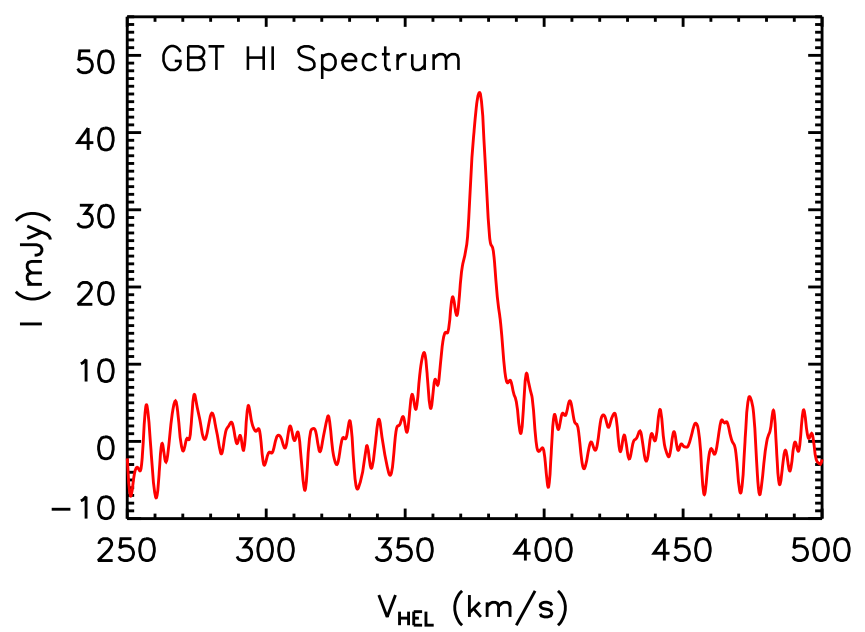

Figure 2. Position-switched, $2 \mathrm{~km} \mathrm{~s}^{-1}$-resolution $\mathrm{H}$ I spectrum obtained with the GBT along the line of sight to Antlia B. The profile's spectral location corresponds to $v_{\text {helio, } \mathrm{HI}}=376 \pm 2 \mathrm{~km} \mathrm{~s}^{-1}$ and the implied $\mathrm{H}$ I properties are consistent with expectations for a gas-rich dIrr satellite of NGC 3109 (which is at $v_{\text {helio }}=403 \mathrm{~km} \mathrm{~s}^{-1}$ ).

\section{PROPERTIES OF ANTLIA B}

\subsection{Distance}

We measure the distance to Antlia B with the TRGB method (e.g., Lee et al. 1993; Rizzi et al. 2007; Crnojević et al. 2014). A Sobel edge detection filter is used to identify the sharp transition in the $r$-band luminosity function that coincides with the brightest, metal-poor RGB stars which can be used as a standard candle. We find $r_{0, \mathrm{TRGB}}=22.55 \pm 0.06 \mathrm{mag}$ for Antlia B, which corresponds to a distance modulus of $\left(m-M_{0}\right)=25.56 \pm 0.16 \mathrm{mag}(D=1.29 \pm 0.10 \mathrm{Mpc})$, after adopting a TRGB absolute value of $M_{r}^{\mathrm{TRGB}}=-3.01 \pm 0.10$ (Sand et al. 2014). This value is identical to the distance to NGC 3109 ( $D=1.28 \pm 0.02 \mathrm{Mpc}$; Dalcanton et al. 2009), to within the uncertainties. Considering both the compatible distance and $\mathrm{H}_{\mathrm{I}}$ velocity between Antlia B and NGC 3109, we conclude that they are associated.

\subsection{Stellar Population}

Inspection of the CMD of Antlia B reveals a complex star formation history (SFH). In Figure 1, we show several representative theoretical isochrones (Dotter et al. 2008 for the 13.5 Gyr stellar populations, and Girardi et al. 2010 for the younger isochrones) over-plotted onto a CMD from within the half light radius, along with several background CMDs drawn from equal areas in outlying regions. The data are consistent with having an old, metal-poor stellar population ( $>10 \mathrm{Gyr}$, $[\mathrm{Fe} / \mathrm{H}] \sim-2)$, with a younger, more metal-rich component ( $\sim 200-400 \mathrm{Myr},[\mathrm{Fe} / \mathrm{H}] \sim-1)$. Given the blue extent of the $\mathrm{RGB}$, there is also room for stellar populations of a few Gyr, although this cannot be disentangled without a full SFH derivation with a CMD-fitting analysis. The younger stellar populations are coincident with relatively heavy foreground star contamination, but the group of stars at $g-r \sim-0.5$ and $r \sim 23.5$ are consistent with a $\sim 100-200$ Myr stellar population, with no counterpart in the background CMDs. In the CMD region occupied by the $\sim 200-400 \mathrm{Myr}$ isochrones, the number of stars in the Antlia B CMD outnumber those seen in the background CMDs by a factor of $\sim 2$. There is no indication of a younger stellar population $(\lesssim 100 \mathrm{Myr})$, which concurs 
Table 1

Antlia B Properties

\begin{tabular}{lc}
\hline \hline Parameter & Value \\
\hline R.A.o $(\mathrm{h}: \mathrm{m}: \mathrm{s})$ & $09: 48: 56.08 \pm 2{ }^{\prime \prime} 1$ \\
Decl.o $_{0}(\mathrm{~d}: \mathrm{m}: \mathrm{s})$ & $-25: 59: 24.0 \pm 3 . ! 8$ \\
$m-M(\mathrm{mag})$ & $25.56 \pm 0.16$ \\
$D(\mathrm{Mpc})$ & $1.29 \pm 0.1$ \\
$M_{V}(\mathrm{mag})$ & $-9.7 \pm 0.6$ \\
$r_{\mathrm{h}}(\operatorname{arcsec})$ & $43.2 \pm 4.2$ \\
$r_{\mathrm{h}}(\mathrm{pc})$ & $273 \pm 29$ \\
$\epsilon$ & $0.30 \pm 0.05$ \\
$\theta(\mathrm{degree})$ & $4.0 \pm 12.0$ \\
$S_{21}\left(\mathrm{Jy} \mathrm{km} \mathrm{s}{ }^{-1}\right)$ & $0.72 \pm 0.05$ \\
$W 50_{\mathrm{H} \mathrm{I}}\left(\mathrm{km} \mathrm{s}^{-1}\right)$ & $17 \pm 4$ \\
$M_{\mathrm{H}}\left(10^{5} M_{\odot}\right)$ & $2.8 \pm 0.2$ \\
$v_{\text {helio, H I }}\left(\mathrm{km} \mathrm{s}^{-1}\right)$ & $376 \pm 2$ \\
\hline
\end{tabular}

with our $\mathrm{H} \alpha$ non-detection. No GALEX imaging is available at the position of Antlia B.

Antlia B's SFH is analogous to that of the original Antlia dwarf (McQuinn et al. 2010), which displays an old, metalpoor stellar population $(\sim 10 \mathrm{Gyr})$, as well as a moderately young component of $\sim 100-400 \mathrm{Myr}$. There is no very young stellar population in Antlia ( $<10 \mathrm{Myr}$ ) in deep HST photometry (McQuinn et al. 2010), and no $\mathrm{H} \alpha$ detection (Lee et al. 2009). Several other so-called "transition" systems (e.g., DDO 210 and LGS 3), have analogous SFHs as Antlia and Antlia B, with no very recent star formation despite the presence of $\mathrm{H}_{\mathrm{I}}$ gas (e.g., Weisz et al. 2011). Upcoming HST observations will provide a clearer picture of the SFH of Antlia B, which will allow for more direct comparisons between the two systems.

\subsection{Stellar Structure and Luminosity}

We determine the structural parameters of Antlia B with the same maximum likelihood technique utilized for Local Group dwarfs (e.g., Martin et al. 2008). Stars which were consistent with old red giant branch isochrones placed at the distance of Antlia B were included in the structural analysis down to $r=24.5 \mathrm{mag}$. We did not explicitly include the younger "blue loop" stellar population in the analysis, although we note in passing that their stellar distribution is more centrally concentrated than the old RGB stars within the central $\sim 15^{\prime \prime}$ of Antlia B. We fit an exponential profile plus a constant background to the data with the central position $\left(\alpha_{0}, \delta_{0}\right)$, position angle $(\theta)$, ellipticity $(\epsilon)$, half-light radius $\left(r_{\mathrm{h}}\right)$ and background surface density as free parameters. Uncertainties on each parameter were calculated via bootstrap resampling the data 1000 times. Careful masking of both the saturated star and background spiral galaxy to the north of Antlia B (Figure 1) ensured that these regions were de-weighted in the maximum likelihood calculation. As a check on our results, we repeated the calculations while only including RGB stars down to $r=23.5$, and found that the derived structural parameters did not change to within the uncertainties; we thus only report the parameters measured from our "deep" maximum likelihood run. The results of the structural analysis are presented in Table 1. Antlia $\mathrm{B}$ has an exponential half-light radius of $r_{\mathrm{h}}=273 \pm 29 \mathrm{pc}$, and an ellipticity of $\epsilon=0.30 \pm 0.05$, both of which are comparable to the MW's classical dwarf spheroidals.

We estimate Antlia B's luminosity directly via aperture photometry (see Sand et al. 2014, 2015). We use an elliptical aperture matched to the half light radius, ellipticity and position angle of Antlia B and randomly place 100 equal area apertures throughout the DECam tile to estimate the background. For the aperture centered on Antlia B itself, we only take flux from the southern half of the ellipse and multiply it by a factor of two, assuming symmetry. This avoids the contamination from the foreground saturated star and background spiral galaxy (Figure 1). Accounting for the fact that our aperture also only covers half the total flux of Antlia B, we find $M_{g}=-9.4 \pm 0.4$ and $M_{r}=-9.9 \pm 0.4$, where the uncertainty was calculated based on the scatter in measurements from the background apertures, as well as the uncertainty in the distance modulus. Using the filter transformations of Jordi et al. (2006), this leads to $M_{V}=-9.7 \pm 0.6 \mathrm{mag}$.

\subsection{H I Content}

We measure the systemic velocity $V_{\text {helio,H I }}$, velocity width at $50 \%$ of the profile peak $W 50_{\mathrm{HI}}$, and the $\mathrm{HI}$ line flux $S_{21}$ for Antlia B from the $\mathrm{H}_{\mathrm{I}}$ spectrum in Figure 2 following the approach detailed in Springob et al. (2005), and tabulate the resulting values in Table 1. Adopting $D=1.29 \pm 0.10 \mathrm{Mpc}$, the $S_{21}$ measurement corresponds to an $\mathrm{H}_{\mathrm{I}}$ mass of $M_{\mathrm{H} \mathrm{I}}=(2.8 \pm 0.2) \times 10^{5} M_{\odot}$, where the uncertainty includes contributions from the $\mathrm{H}_{\mathrm{I}}$ spectrum and TRGB distance added in quadrature. The $\mathrm{H}$ I mass to stellar luminosity ratio of Antlia B is therefore $M_{\mathrm{H}} / L_{V} \sim 0.4\left(M_{\odot} / L_{\odot}\right)$, implying that Antlia B is a gas-rich dwarf irregular galaxy. We compare Antlia B's H I gas fraction to other Local Volume dwarf irregulars in Section 4.

\section{DISCUSSION AND CONCLUSIONS}

We have presented the discovery of Antlia B, a faint dwarf galaxy $R \sim 72 \mathrm{kpc}$ in projection from the center of NGC 3109 $\left(D=1.28 \mathrm{Mpc} ; v_{\odot}=403 \mathrm{~km} \mathrm{~s}^{-1}\right)$. Antlia B's inferred distance $(1.29 \pm 0.10 \mathrm{Mpc})$ and $\mathrm{H}_{\mathrm{I}}$ velocity $\left(v_{\odot}=376 \mathrm{~km} \mathrm{~s}^{-1}\right)$ make the physical association with NGC 3109 certain. The stellar population of Antlia B is complex, with an old and metal-poor component $(\gtrsim 10 \mathrm{Gyr}$; $[\mathrm{Fe} / \mathrm{H}] \sim-2)$ as well as a younger stellar population $(\sim 200-400 \mathrm{Myr})$. We detect $M_{\mathrm{H}_{\mathrm{I}}}=(2.8 \pm$ $0.2) \times 10^{5} M_{\odot}$ of $\mathrm{H}$ I gas in Antlia B, implying that it is a gasrich dwarf irregular galaxy.

We compare the physical properties of Antlia B with other members of the NGC 3109 association and Local Volume in Figure 3. To compute the $\mathrm{H}$ I gas mass to stellar mass ratio (which probes the efficiency of gas conversion to stars) we have assumed that $\left(M_{\text {star }} / L_{V}\right) /\left(M_{\odot} / L_{\odot}\right)=1$ and translated our absolute $V$-band magnitudes. Antlia B's properties are broadly consistent with other Local Volume members of its size and luminosity. In particular, it is most similar to Leo P (McQuinn et al. 2013), albeit with a slightly lower $\mathrm{H}_{\mathrm{I}}$ gas mass fraction.

Given their proximity to NGC 3109, both Antlia (projected separation of $\sim 40 \mathrm{kpc}$ ) and Antlia B (projected separation of $\sim 70 \mathrm{kpc}$ ) may be surprisingly gas-rich. Galaxies with similar stellar masses to NGC $3109\left(M_{\text {star }} \sim 1 \times 10^{8} M_{\odot}\right)$ in the ELVIS simulation suite (Garrison-Kimmel et al. 2014) have dark matter halo virial radii of $\sim 100 \mathrm{kpc}$. Therefore, unless the line of sight separations of Antlia and Antlia B are at least as large as their projected ones (and keeping in mind that both Antlia and Antlia B's line of sight distance uncertainty is $\sim 100 \mathrm{kpc}$ ), they lie within the virial radius of their parent galaxy. Satellites within the MW's virial radius with similar 

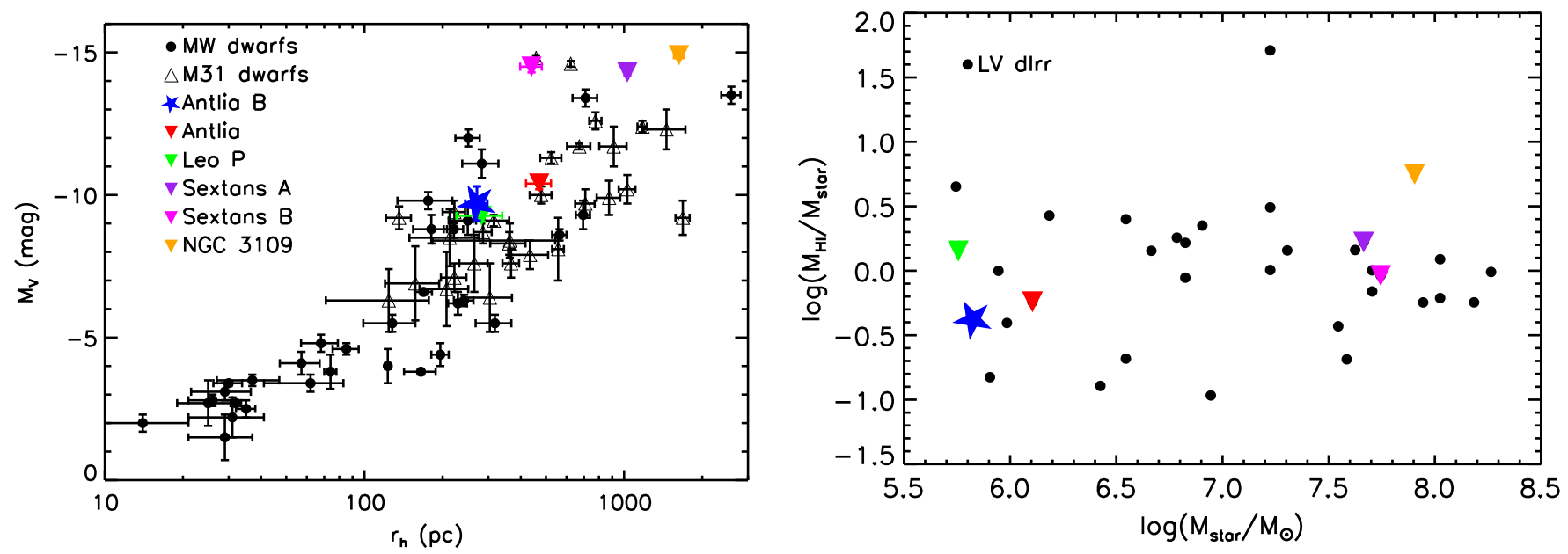

Figure 3. Left: absolute magnitude as a function of half light radius of Antlia B and the rest of the NGC 3109 association. Also plotted are Local Group dwarf galaxies (McConnachie 2012; Sand et al. 2012; Koposov et al. 2015). Antlia B is similar to Leo P, while the NGC 3109 association as a whole is consistent with the dwarfs in the Local Group. Right: the ratio of $M_{\mathrm{H}} / M_{\text {star }}$ as a function of stellar mass for the NGC 3109 association and Local Volume dwarf irregulars. Antlia B has a typical $M_{\mathrm{H}} / M_{\text {star }}$ for gas rich, low stellar mass members of the Local Volume.

stellar masses to Antlia and Antlia B are devoid of H I (Spekkens et al. 2014), consistent with a scenario in which ram pressure stripping from the MW's hot gas corona has swept away their interstellar media during past pericentric passages (e.g., Gatto et al. 2013), although other physical mechanisms may contribute to this gas removal (e.g., Grcevich \& Putman 2009; Spekkens et al. 2014 for recent discussions). The $\mathrm{H}_{\mathrm{I}}$ richness of Antlia and Antlia B therefore suggests that they have not yet reached a point in their orbits where ram pressure stripping is effective: this could be because they have not yet had a pericentric passage, or because either their orbital speeds are not high enough or NGC 3109's hot corona is not dense enough at pericenter for the mechanism to have much effect.

Recently it has been suggested that the NGC 3109 dwarf association displays a linear configuration in both spatial and velocity coordinates, suggestive of an infalling filament or tidal origin (Bellazzini et al. 2013; Pawlowski \& McGaugh 2014). While Antlia B does appear to fall on this proposed linear structure, this is necessarily the case because of the geometry of our survey, which extended out to $R \sim 100 \mathrm{kpc}$ from NGC 3109.

The substructure properties of sub-MW mass halos is an unobserved portion of parameter space, but simulations suggest that they will have a wealth of substructure (e.g., Wheeler et al. 2015). The discovery of Antlia B, and upcoming work on NGC 3109's overall substructure properties, will provide tests of the $\Lambda+\mathrm{CDM}$ picture of galaxy formation in a new mass regime.

D.J.S. acknowledges support from NSF grant AST-1412504. K.S. acknowledges support from the Natural Sciences and Engineering Research Council of Canada. B.W. and J.H. were supported by an NSF Faculty Early Career Development (CAREER) award (AST-1151462). The National Radio Astronomy Observatory is a facility of the National Science Foundation operated under cooperative agreement by Associated Universities, Inc. This work was supported in part by National Science Foundation grant No. PHYS-1066293 and the Aspen Center for Physics. This project used data obtained with the Dark Energy Camera, which was constructed by the Dark
Energy Survey collaboration. Funding for the DES Projects has been provided by the DOE and NSF(USA), MISE(Spain), STFC(UK), HEFCE(UK). NCSA(UIUC), KICP(U. Chicago), CCAPP(Ohio State), MIFPA(Texas A\&M), CNPQ, FAPERJ, FINEP (Brazil), MINECO(Spain), DFG(Germany) and the collaborating institutions in the DES, which are Argonne Lab, UC Santa Cruz, University of Cambridge, CIEMAT-Madrid, University of Chicago, University College London, DES-Brazil Consortium, University of Edinburgh, ETH Zurich, Fermilab, University of Illinois, ICE (IEEC-CSIC), IFAE Barcelona, Lawrence Berkeley Lab, LMU Munchen and the associated Excellence Cluster Universe, University of Michigan, NOAO, University of Nottingham, Ohio State University, University of Pennsylvania, University of Portsmouth, SLAC National Lab, Stanford University, University of Sussex, and Texas A\&M University.

\section{REFERENCES}

Arraki, K. S., Klypin, A., More, S., \& Trujillo-Gomez, S. 2014, MNRAS, 438, 1466

Bechtol, K., Drlica-Wagner, A., Balbinot, E., et al. 2015, ApJ, 807, 50

Bellazzini, M., Oosterloo, T., Fraternali, F., \& Beccari, G. 2013, A\&A, 559, L11

Bovy, J., \& Dvorkin, C. 2013, ApJ, 768, 70

Clemens, J. C., Crain, J. A., \& Anderson, R. 2004, Proc. SPIE, 5492, 331

Crnojević, D., Sand, D. J., Caldwell, N., et al. 2014, ApJL, 795, L35

Dalcanton, J. J., Williams, B. F., Seth, A. C., et al. 2009, ApJS, 183, 67

Deason, A. J., Wetzel, A. R., Garrison-Kimmel, S., \& Belokurov, V. 2015, MNRAS, 453, 3568

D’Onghia, E., \& Lake, G. 2008, ApJL, 686, L61

Dotter, A., Chaboyer, B., Jevremović, D., et al. 2008, ApJS, 178, 89

Flaugher, B., Diehl, H. T., Honscheid, K., et al. 2015, ApJ, submitted (arXiv:1504.02900)

Garrison-Kimmel, S., Boylan-Kolchin, M., Bullock, J. S., \& Lee, K. 2014, MNRAS, 438, 2578

Gatto, A., Fraternali, F., Read, J. I., et al. 2013, MNRAS, 433, 2749

Giovanelli, R., Haynes, M. P., Adams, E. A. K., et al. 2013, AJ, 146, 15

Girardi, L., Williams, B. F., Gilbert, K. M., et al. 2010, ApJ, 724, 1030

Grcevich, J., \& Putman, M. E. 2009, ApJ, 696, 385

Hargis, J. R., Willman, B., \& Peter, A. H. G. 2014, ApJL, 795, L13

Jordi, K., Grebel, E. K., \& Ammon, K. 2006, A\&A, 460, 339

Kennicutt, R. C., Jr., Lee, J. C., Funes, José G. , S. J., et al. 2008, ApJS, 178,247

Kim, D., \& Jerjen, H. 2015, ApJL, 808, L39 
Koposov, S. E., Belokurov, V., Torrealba, G., \& Evans, N. W. 2015, ApJ, 805,130

Laevens, B. P. M., Martin, N. F., Bernard, E. J., et al. 2015a, ApJ, submitted (arXiv:1507.07564)

Laevens, B. P. M., Martin, N. F., Ibata, R. A., et al. 2015b, ApJL, 802, L18

Lee, J. C., Gil de Paz, A., Tremonti, C., et al. 2009, ApJ, 706, 599

Lee, M. G., Freedman, W. L., \& Madore, B. F. 1993, ApJ, 417, 553

Lewis, P. M., Rogers, H., \& Schindler, R. H. 2010, Proc. SPIE, 7735 , $77353 \mathrm{C}$

Macciò, A. V., Kang, X., Fontanot, F., et al. 2010, MNRAS, 402, 1995

Martin, N. F., de Jong, J. T. A., \& Rix, H.-W. 2008, ApJ, 684, 1075

Martin, N. F., Ibata, R. A., McConnachie, A. W., et al. 2013, ApJ, 776, 80

Martin, N. F., Nidever, D. L., Besla, G., et al. 2015, ApJL, 804, L5

McConnachie, A. W. 2012, AJ, 144, 4

McQuinn, K. B. W., Skillman, E. D., Berg, D., et al. 2013, AJ, 146, 145

McQuinn, K. B. W., Skillman, E. D., Cannon, J. M., et al. 2010, ApJ, 721, 297

Pawlowski, M. S., \& McGaugh, S. S. 2014, MNRAS, 440, 908

Peñarrubia, J., Pontzen, A., Walker, M. G., \& Koposov, S. E. 2012, ApJL, 759, L42
Pontzen, A., \& Governato, F. 2012, MNRAS, 421, 3464

Rizzi, L., Tully, R. B., Makarov, D., et al. 2007, ApJ, 661, 815

Sand, D. J., Crnojević, D., Bennet, P., et al. 2015, ApJ, 806, 95

Sand, D. J., Crnojević, D., Strader, J., et al. 2014, ApJL, 793, L7

Sand, D. J., Strader, J., Willman, B., et al. 2012, ApJ, 756, 79

Schlafly, E. F., \& Finkbeiner, D. P. 2011, ApJ, 737, 103

Schlegel, D. J., Finkbeiner, D. P., \& Davis, M. 1998, ApJ, 500, 525

Spekkens, K., Urbancic, N., Mason, B. S., Willman, B., \& Aguirre, J. E. 2014, ApJL, 795, L5

Springob, C. M., Haynes, M. P., Giovanelli, R., \& Kent, B. R. 2005, ApJS, 160,149

Stetson, P. B. 1994, PASP, 106, 250

Tully, R. B., Rizzi, L., Dolphin, A. E., et al. 2006, AJ, 132, 729

Valdes, F., Gruendl, R. \& DES Project. 2014, in ASP Conf. Ser. 485, Astronomical Data Analysis Software and Systems XXIII, ed. N. Manset \& P. Forshay (San Francisco, CA: ASP), 379

Weisz, D. R., Dalcanton, J. J., Williams, B. F., et al. 2011, ApJ, 739, 5

Wheeler, C., Onorbe, J., Bullock, J. S., et al. 2015, MNRAS, 453, 1305

Willman, B. 2010, AdAst, 2010, 285454 